\title{
On dense subspaces of Moore spaces II
}

by

Ben Fitzpatrick, Jr. (Auburn, Ala.)

It follows from Theorem 1 of this paper that if $S$ is a normal Moore space with no dense metric subspace then $S$ is of Type D in the sense of Bing [1].

A development for a Moore space $S$ is a sequence satisfying Axiom $\mathbf{1}_{\mathbf{3}}$ of [3]. If $G$ is a development for $S$, then $C(G)$ denotes the set of all points $x$ of $S$ such that, if $R$ is a region containing $x$, there exists a positive integer $n$ such that every element of $G(n)$, the $n$th term of $G$, intersecting an element of $G(n)$ containing $x$ is a subset of $R$.

THEOREM 1 . If $S$ is a normal Moore space which is not a counterexample of Type $\mathrm{D}$ and $M$ is a subset of $S$ then there exists a development $G$ for $S$ such that $C(G)$ is dense in $S$ and $C(G) \cdot M$ is dense in $M$.

Proof. Suppose that $S$ is as in the hypothesis, $H$ is a development of $S$, and $M C$ is a subset of $S$. Using a well-ordering argument, one can construct a sequence $K$ such that

(1) for each $i$ no region of $H(i)$ contains two points of $K(i)$,

(2) if $p$ is a point of $M$ some region of $H(i)$ contains $p$ and a point of $M \cdot K(i)$,

(3) if $p$ is a point of $S$ some region of $H(i)$ contains $p$ and a point of $K(i)$, and

(4) $K(i)$ is a subset of $K(i+1)$.

It is clear that each term of $K$ is a closed and isolated point set, that $\sum_{i} K(i)$ is dense in $S$, and that $M \cdot \sum_{i} K(i)$ is dense in $M$. By hypothesis, for each $i$ there is a collection of mutually exclusive regions covering $K(i)$, each region of the collection containing only one point of $K(i)$. Using normality, one can obtain a discrete collection of mutually exclusive regions covering $K(i)$ each containing only one point of $K(i)$, and, as regions covering of the proof of Theorem 5 of [2], $K(i)$ has Younglove's Property Q [5], so that some development satisfies Axiom $O$ of [4] at each point of $K(i)$. It follows that some development satisfies Axiom $\mathbf{C}$ at each point of $\sum_{i} K(i)$. 
CoRolLART 1. If $S$ is a normal Moore space which contains no dense metric subspace, then $S$ is a counterexample of Type $\mathrm{D}$.

Proof. This follows from Theorem 1 and from Moore's metrization. theorem [4].

COROLLARY 2. If $S$ is a normal, nonmetrizable Moore space which is not a counterexample of Type $\mathrm{D}$, then $S$ contains a dense, nonmetrizable subspace $S^{\prime}$ such that $S=D+M$, where $D$ is a metrizable domain dense in $S^{\prime}, M$ is the boundary in $S^{\prime}$ of $D$.

Pro of. Since $S$ is not metrizable, there exists [1] a discrete collection $I$ of mutually exclusive closed point sets not satisfying the definition of collectionwise normality with respect to any collection of domains. Denote by $I^{\prime}$ the collection to which $M^{\prime}$ belongs if and only if, for some $M$ in $I$, $M^{\prime}$ is $M$-Int $M$ ( $M$ minus its interior). Then $I^{\prime}$ is a discrete collection of closed sets not satisfying the definition of collectionwise normality with respect to any collection of domains, and $I^{\prime *}$ is closed and nowhere dense. From Theorem 1 there exists a development $G$ for $S$ such that $O(G)$. $\left(S-I^{\prime *}\right)$ is dense in $S-I^{\prime *}$ and thus in $S$. Let $M=I^{\prime *}, D=(S-M)$.

$(C)$, and $S^{\prime}=D+M$. Then $D, M$, and $S^{\prime}$ have the desired properties.

\section{References}

[1] R. H. Bing, A translation of the normal Moore space conjecture, Proc. Amer. Math. Soc. 16 (1965), pp. 612-619.

[2] B. Fitzpatrick, On dense subspaces of Moore spaces, Proc. Amer. Math. Soc. 16 (1965), pp. 1324-1328.

[3] R. L. Moore, Foundations of point set theory, Amer. Math. Soc. Coll. Publ. 13, Revised Edition. (Providence, 1962).

[4] - A set of axioms for plane analysis situs, Fund. Math. 25 (1935), pp. 13-28. [5] J. N. Younglove, On dense metric subspaces of certain nonmetric spaces, Fund. Math. 48 (1959), pp. 15-25.

AUBURN UNIVERSITY

Auburn, Alabama

\section{A complete, infinitary axiomatization of weak second-order logic}

by

E. G. K. Lopez-Escobar (College Park, Maryland)

0. Introduction. One of the reasons (amongst others) why secondorder $\operatorname{logic}(\mathrm{s})$ are studied is that it is possible to characterize (up to isomorphism) many mathematical structures. However for certain structures, e.g. the natural numbers, archimidean ordered fields, a logic weaker tures, e.g. the natural numbers, (hence the name weak second-order) suffices. In the weak second-order theories the second-order variables are understood as ranging over finite, non-empty sequences of those objects to which the first-order variables refer. The notion of consequence which is customarily used in weak second-order theories is a semantical (model-theoretic) one. As weak second-order theories is a sothods of Gödel [G] can be used to show that, as long as proofs are required to be of finite length, no complete formalization can be obtained for weak second-order logic. In this paper we present a formalization in which the proofs are of infinite length and which is complete. Furthermore, all but one of the rules of inference have finitely many premises and the rule with infinitely many premises is similar to the $\omega$-rule (Carmap's rule). ${ }^{1}$ )

In the last section we comment briefly on how to obtain a more constructive axiomatization.

1. The weak second-order language. The language may be briefly defined as follows:

1.1. Symbols.

(a) Individual variables: $v_{0}, \ldots, \nabla_{n}, \ldots$

(b) Individual constants: $\mathrm{c}_{0}, \ldots, \mathrm{c}_{n}, \ldots$.

(c) Second-order variables: $\nabla_{0}, \ldots, V_{n}, \ldots$.

(d) Predicate symbol (binary): $P$.

(1) The author is indebted to Professor Mostowski for raising the problem of finding a complete axiomatization for weak second-order logic. 